\title{
Synthetic biology: a new approach to study biological pattern formation
}

\author{
Chenli Liu ${ }^{1,3}$, Xiongfei $\mathrm{Fu}^{2}$ and Jian-Dong Huang ${ }^{1, *}$ \\ 1 Department of Biochemistry, The University of Hong Kong, Hong Kong, China \\ 2 Department of Physics, The University of Hong Kong, Hong Kong, China \\ ${ }^{3}$ Center for Synthetic Biology Engineering Research, Guangzhou Institute of Advanced Technology, Chinese Academy of \\ Sciences, Guangzhou 511458, China \\ * Correspondence: jdhuang@hku.hk
}

Received October 11, 2013; Accepted November 15, 2013

\begin{abstract}
The principles and molecular mechanisms underlying biological pattern formation are difficult to elucidate in most cases due to the overwhelming physiologic complexity associated with the natural context. The understanding of a particular mechanism, not to speak of underlying universal principles, is difficult due to the diversity and uncertainty of the biological systems. Although current genetic and biochemical approaches have greatly advanced our understanding of pattern formation, the progress mainly relies on experimental phenotypes obtained from timeconsuming studies of gain or loss of function mutants. It is prevailingly considered that synthetic biology will come to the application age, but more importantly synthetic biology can be used to understand the life. Using periodic stripe pattern formation as a paradigm, we discuss how to apply synthetic biology in understanding biological pattern formation and hereafter foster the applications like tissue engineering.
\end{abstract}

\section{INTRODUCTION}

Biological systems often form intricate spatiotemporal patterns, such as zebra stripes, peacock feathers, leopard spots, fish scales, flower petals etc. The biological process that generates these ordered structures is termed "pattern formation"[1]. It usually involves changes in gene expression, cell movement, proliferation, and cell death, intricately coordinated by multiple signaling mechanisms. Pattern formation is a common interest of molecular biologists, developmental biologists, synthetic biologists, tissue engineers, system biologists, and theoretical biologists. Understanding how these patterns arise from the coordinated molecular responses is an outstanding challenge to generations of scientists.

In the past decade, the synthetic biologists have made great advance in bringing concepts of physics and engineering into biology. Engineering cells to have novel functions [2-7] is the major aim of synthetic biology. However, the other important aim of this field, "build life to understand it," is increasingly emphasized $[8,9]$. Biological patterns are traditionally studied via either forward or reverse genetic approach. Applying synthetic biology in investigation of principles of biological pattern formation then would be a paradigm shift. In this review, we will focus on periodic biological patterns.

\section{PERIODIC BIOLOGICAL PATTERNS AND EXPERIMENTAL INVESTIGATION}

The biological patterns anatomically often have repeated units. For example man has 10 fingers, 24 ribs, and 32 teeth. A spatial "periodic" pattern typically comprises repeated units separated by orderly intervals. People can easily see this periodicity in the patterns of zebra stripes, peacock feathers, fish scales, Drosophila segments, and vertebrate embryo somites. Periodicity also takes place temporally, such as the contraction of our heart muscle, and the oscillation of the harmonic motion [10].

The segmentation of the fruit fly Drosphila melanogaster is one of the major paradigms in the study of periodic pattern. In the past three decades, about 40 genes have been identified in D. melanogaster in the segmentation process. These genes act in a cascade progressively resulting in the subdivision of the anteroposterior (AP) 
axis $[11,12]$ (see Figure 1 for details).

Somitogenesis, as an important developmental process of vertebrate embryo, has been extensively studied $[14,15]$. Somites are segments in embryo, particularly blocks of cells surrounding a cavity structure [14]. The somite appears rhythmically from the presomitic mesoderm (PSM). PSM are two parallel bands of mesenchymal tissue lying alongside the neural tube [14]. With a temporal interval of $30 \mathrm{~min}$ in zebrafish, $90 \mathrm{~min}$ in chicken, $120 \mathrm{~min}$ in mice, and around $4 \mathrm{~h}$ in humans, somites are generated periodically along the AP axis [16]. Later on, these somites can develop into skeletal muscles, dermis, and vertebrae. In past decades, much effort has been made to uncover the pacemaker that drives the oscillation of the cyclic gene expression. In chick embryo, it has been shown that fibroblast growth factor 8 (FGF8)
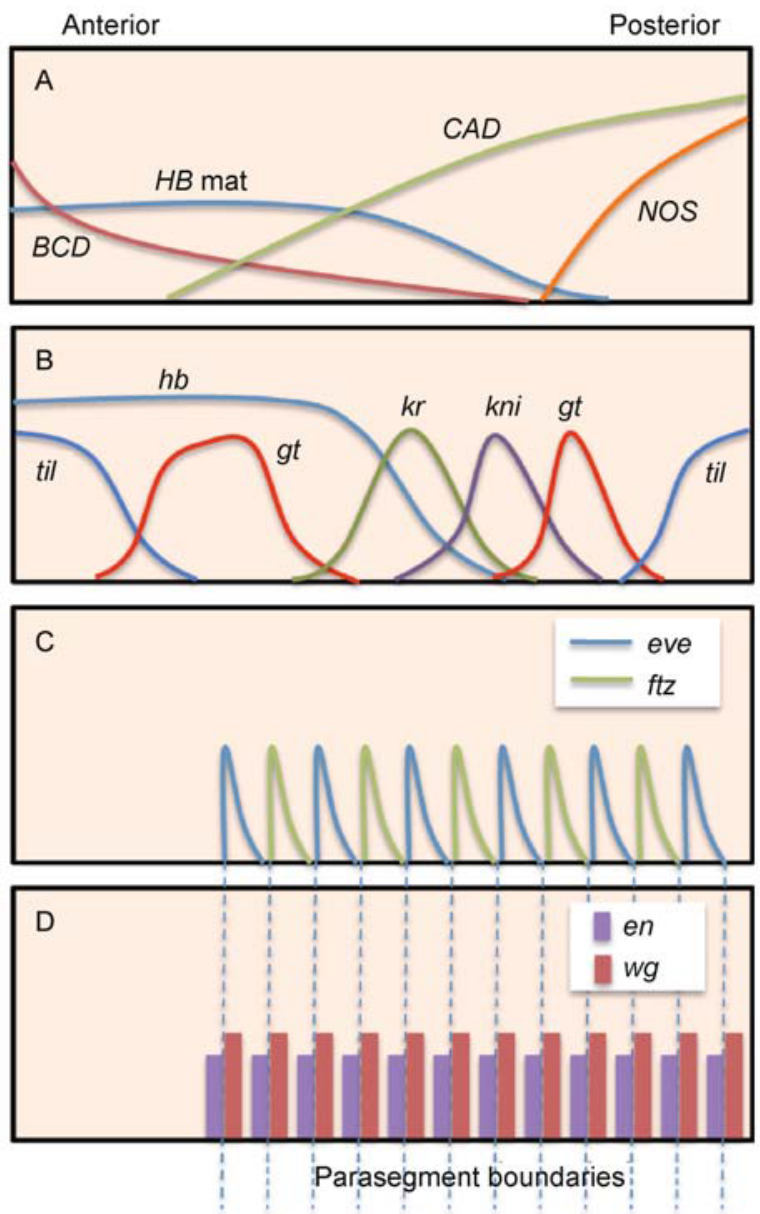

Figure 1. Drosophila melanogastersegmentation. (A) Maternal signals. A "ready mix" cytoplasm has been prepared during the oogenesis. In the cytoplasm of the egg, the matemal transcripts of hunchback $(h b)$ and caudal (cad) are uniformly distributed; the transcript of bicoid $(b c d)$ is mainly localized at the anterior pole, while a protein and RNA complex is localized at the posterior of the egg. After fertilization, these maternal transcripts are translated rapidly, generating protein gradients along the egg. (B) Gap domains. Signals embedded in the egg-shell activate Torso, a transmembrane receptor localized at the poles of the egg. Meanwhile, at specific positions along the AP axis of the egg, a group of zygotic "gap" genes, e.g., tailless (tll), giant ( $g t)$, Kruppel (Kr), are activated by the signals from maternal proteins. (C) Pair-rule pattem. Although the expression profile of the gap genes is not periodic, the interaction of their transcriptional factor products and signals from maternal proteins provides nuclei a specific axial identity. This identity is thereafter interpreted by the pair-rule genes, e.g., hairy ( $h$ ), runt (run) and even skipped (eve), generating periodic gene expression. Further transcriptional interaction between pair-rule genes and their downstream genes (e.g., fushi tarazu $(f t z))$ refine the expression stripes. The stripe boundaries predict the parasegment boundaries. (D) Segment polarity pattern. Despite that the gene expression of pair-rule genes is transient, once cell boundaries form, the pair-rule proteins in each parasegment activates the segment polarity genes, whose expression is stable. Some genes like engrailed (en) is stable even in the adult. Parasegment boundaries form between the cells expressing en and wingless $(w g)$, respectively. Later on, the segment boundaries are generated based on the parasegments. Adapted from Peel et al., 2005 [13]. 
is involved in the translation of clock signal into segments [17]. The mRNA level of $f g f 8$ is high in posteriormost PSM and progressively decreases toward anteriormost side, forming a gradient over the PSM (Figure 2). Overexpression of $f g f 8$ blocked the segmentation and kept the PSM cells in an immature state, indicating that the expression level of FGF8 correlates with the fate of PSM cells. Thus, it is proposed that there is a threshold level of FGF8, termed the "determination front" (Figure 2). As the elongation of the body axis, the determination front recedes, allowing the cells enter the segmentation process. Thus, the distance of boundaries of the somites probably is set by the period of the oscillation.

\section{Numerical constancy}

Another fascinating question is how precise numbers of modules/elements/body units are achieved in periodic patterns, such as 10 fingers, 24 ribs, 32 teeth, and 33 vertebrae in humans. What mechanisms determine the number and guarantee the constancy? Held postulated that there is a "halt condition" to stop the repetition of the pattern element [10]. If the "halt condition" were not functional, the pattern element would repeat ceaselessly. Actually, such 'infinite' mutants have been increasingly observed. For instance, in Caenorhabditis elegans, unc86 mutations caused the cell lineages repeat many times [19]; in Drosophila, bag-of-marbles mutations increased the cell number of ovarian cyst from 16 to hundreds [20]; in Antirrhinum, floricaula mutation made flowers become repeated meristems [21].

It is intuitive that, given a constraint of element size, the number of the pattern element could be controlled by the body size. In alligator, there are more stripes on the back of the male alligator due to their longer body size [22]. On the contrary, the element number can be independent of body size, e.g., the somite number in Xenopus [23].

\section{MODELS FOR PERIODIC PATTERN}

"I am never content until I have constructed a mechanical model of the subject I am studying. If I succeed in making one, I understand. Otherwise, I do not." - Lord Kelvin Mathematic models are essential. They can integrate experimental data; extract the key components; predict the results upon genetic or physical perturbations. It's a highly intellectual challenge to summarize experimental data at various levels into a mathematic model. So far they have two major forms: i) analyze every component and simulate every interaction in a system [24]; ii) omit complicated details of a system but extract its nature [25]. The first type of model is used to describe relatively simple systems. If the system were complex, e.g., involving spatiotemporal dynamics, it would be difficult to make a thorough analysis and develop a plausible "detailed" model.

A great diversity of models have been suggested for the periodic patterning phenomena [10]. As Murray drummed in his book [22], these models are not the actual mechanisms of the natural pattern. Similarly, Wolpert shared his Maxim: "do not infer process from pattern, since so many processes can produce the same pattern" [26]. Here, we briefly introduce two important models of theirs.

\section{Reaction-diffusion model}

Reaction-diffusion or Turing model is one of the most well-known mathematic models for pattern formation [27]. Alan Turing, one of the founders of modern computer [28], proposed this brilliant model in a seminal paper [29]. Intuitively, people would believe that diffusion always contributes to stabilize the system. But Turing found, in presence of diffusion, it is possible that the origin locally stable state of a reaction system becomes

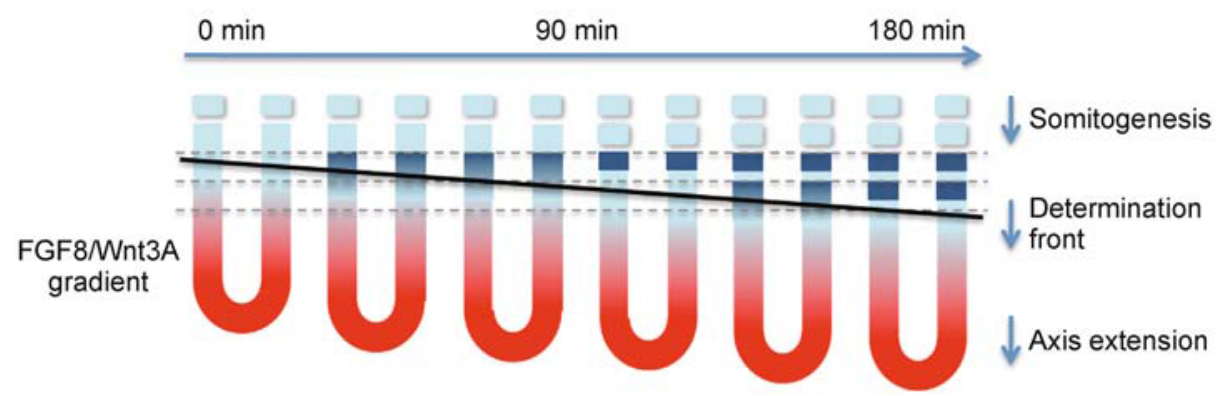

Figure 2. Model of segmentation in mouse vertebrates. The FGF8/Wnt3A gradient is shown in red. The expression of Mesp2/ c-meso1 is shown in blue. The determination front is shown as a black line. Adapted from Pourquie, 2003 [18]. 
unstable. Mathematically, the coupling of diffusion and reaction may make small perturbations on the homogenous stable state amplified. The global instabilities, together with the boundary condition, can determine the periods of the patterns. In other words, the diffusion and reaction of the molecules leads to the formation of peak and trough of the molecular concentration (Figure 3A). These molecules are termed as morphogens. And their distribution profiles will be the prepattern to develop. It differs from the prepattern models in the reaction term, which produces the morphogens [27].

The reaction-diffusion model has been considered to be the standard in the mathematic models for biological pattern formation [27]. However, in the experimental biology community, this model is not widely accepted. Fitzhugh and Nagumo proposed an important derivative of Turing model to translate the mathematic language into the one biologists use [30]. It is an activator-inhibitor excitable system, such as the neuron. In this twocomponent system, the production of both components is activated by one of the chemicals, while the other one plays a role to inhibit the growth of the system. With proper parameters and under certain conditions, the model can produce plain wave, rotating spirals, ring wave, stripe wave, stationary localized pulse, et al. Furthermore, to get a stable spatial pattern, Gierer and Meinhardt proposed that a Turing pattern based on lateral inhibition only requires a short-range positive feedback and a long-range negative feedback [31], indicating that a faster diffusion of the inhibitor and a slower diffusion of the activator. These refinements make the general principle of Turing model much easier to be applied in complex systems. It has been shown that many systems may satisfy this condition and are capable to form Turing Patterns, such as chemotactic cell migration [32], neuronal interactions [33], mechanochemical activity [34] and so on. However, it's hard to directly demonstrate the Turing's model in a real biological system. As Gierer and Meinhardt suggested, the diffusion coefficient of activator should be much slower than that of inhibitor [31,35]. But people always lack of direct evidence on the diffusivity difference in a real biologic system, due to the difficulties to characterize the exact activator/inhibitor. Until recently, a group of scientists succeeded to measure the diffusivity of GFP fused Nodal and Lefty, an activator/ inhibitor-based system during zebrafish embryogenesis by fluorescence recovery after photobleaching method [36].

A
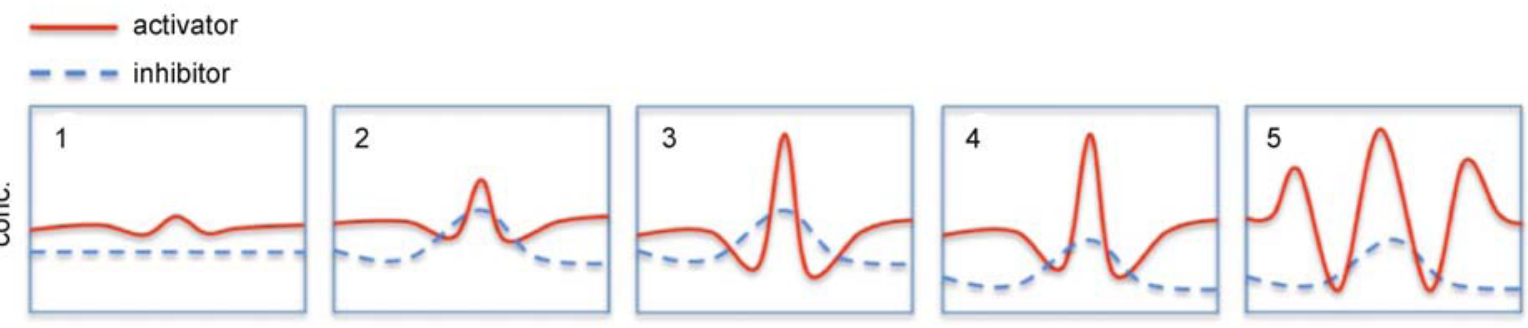

B
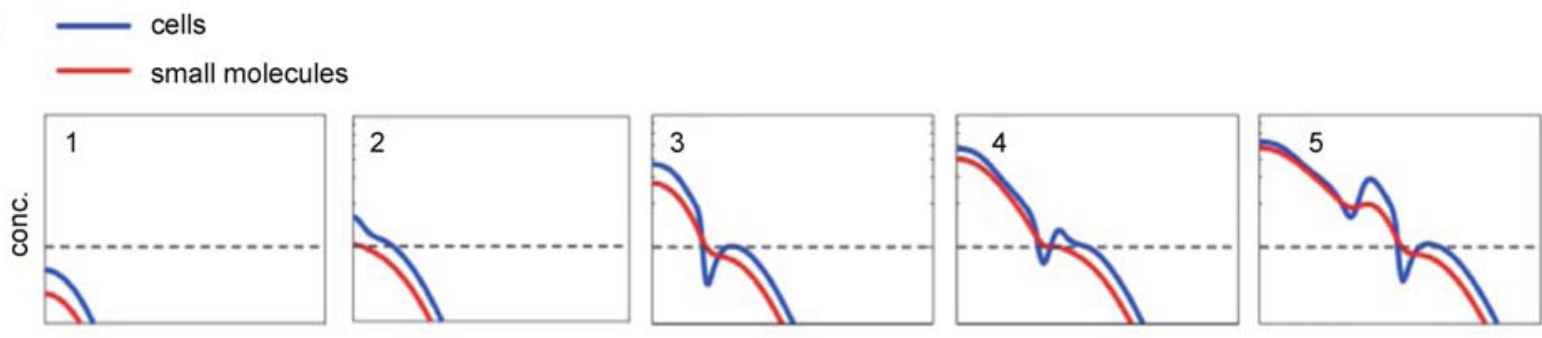

Figure 3. Scheme of the formation of Turing or density-dependent motility patterns. (A) Turing pattern. 1, The initial condition. The concentration of the activator is supposed to be higher than that of inhibitor. 2, The concentration of activator increases at the center due to the self-enhancing property. 3, The concentration of inhibitor increase. Due to the higher diffusion rate, inhibitor move laterally, leading to the decrease of the concentration of activator at those regions. 4 , The decrease of activator concentration in turn results in the decrease of inhibitor. 5, For the outer region, the low concentration of inhibitor causes the increase of activator. This initiates the new peak of activator. Adapted from Kondo and Miura, 2010 [27]. (B) Density-dependent motility pattern. 1, The initial wave front starts to propagate as a Fisher's wave front. Both the cell density and density signal molecule concentration is low. 2 , The density signal molecule reaches the threshold, while the cell motility in this region drops. A "bud" merges, due to the effective limited diffusion. 3, The bud amplifies to a "mound," separated from the frontal region by a "cleft," while the front continues propagating. 4 , The front reaches the motility threshold again, generating a new "bud." 5 . The new "bud" grows to a "mound," while the front keeps repeating the same process. 


\section{The density-dependent motility model}

Physicists also proposed that density-dependent motility could be an alternative mechanism to form periodical patterns. Cates et al. studied the model, in which the motility of the bacteria depends on the local cell density. Using stability analysis, they found the system could form stable patterns with small random fluctuations around the equilibrium cell density. However, to balance the divergence of the high frequency mode of the instability, they need introduce a forth order term [31]. Another more experimentally relevant density-dependent motility model was proposed, which includes a density signaling field $[38,39]$. Instead of instability around the equilibrium, people found this density-dependent motility model could generate periodical pattern as the density of leading front reaches the motility threshold during the propagation. This mechanism is different from typical reaction diffusion model, and has been verified through a synthetic biological system. This model would also be applied to a system with two diffusive chemicals, in which the diffusivity of one depends on the other chemical that indicates the local concentration.

\section{The clock and wavefront model}

The clock and wavefront model was proposed for somitogenesis. It comprises of two components: an oscillating two-state "clock" and a unidirectionally propagating wave [40]. In general, all cells synchronously oscillate between two states. Once the front of the traveling wave reaches, the oscillation stops. As the wave sweeps over the developing zone, a periodic pattern with alternating states is left behind.

\section{SYNTHETIC BIOLOGY}

Synthetic biology is a new and fast growing field that seems still has no widely accepted definition. Some people define synthetic biology as "the design and construction of new biological parts, devices and systems, and the re-design of existing natural biological systems for useful purposes" (http://syntheticbiology. org/). Generally, these novel functions were achieved by introducing new genetic networks termed "genetic circuits."

In the past decade, synthetic biology has been applied to engineer new functions [41,42], such as population controlling [6], cellular counter [43], "photoprinter" [4], edge detector [3], and so on. The other important aspect of synthetic biology is to understand the life via an engineering approach $[8,9]$. Various biological phenomena have been studied, including biological oscillator $[2,7]$, bacterial two-component system [44], band detection[5], biofilm development [45], rebuilt ecosystem [46], antibiotic resistance [47], etc.

\section{Pattern formation with synthetic biology approaches}

Basu et al. engineered a "band-pass" filter by separating luxRI system into sender (expressing luxI and producing $\mathrm{N}$-acylho-moserine lactone (AHL)) and receiver cells (expressing luxR and responding to the AHL gradient). They designed an ingenious genetic circuit to make cells respond differently to the different level of AHL, resulting in a bull-eye pattern in solid agar. Here, sender cells constitutively produced diffusible AHL. With certain production and decay rate, AHL formed a gradient in solid agar. Receiver cells containing a series of genetic elements (e.g., luxR, $c I$, and $l a c l$ ) were in advance mixed in the agar. They only expressed GFP when AHL concentration was in a narrow range. Three LuxR mutants with different sensitivities to AHL were applied to generate various patterns [5]. With the synthetic genetic circuit, they directly demonstrated the pattern formation mechanism of morphogen gradient.

More recently, Danino et al. used luxRI system to synchronize $E$. coli population, created a genetic oscillator [2]. Compared with previous synthetic oscillation systems, their design was simple but led to beautiful temporally periodic patterns. They placed $l u x I$, a reporter yemGFP and aiiA (AHL-degrading enzyme) under the control of $P_{l u x}$. LuxR-AHL complex induced aiiA expression, which in turn negatively regulated the expression of luxI. With the aid of microfluidics devices and fluorescent microcopy, the bulk oscillation was temporally recorded. Moreover, the period and amplitude of the oscillator could be tuned by controlling flow rate in the microfluidics.

Liu et al. engineered bacterial cells to explore a novel principle of periodic pattern formation [39]. They constructed a model system containing the basic ingredients of growth, motility and cell-to-cell signaling for pattern formation. A simple regulatory interaction, involving the inhibition of cell motility at high cell densities, was introduced by controlling the chemotaxis circuit of $E$. coli by a heterologous quorum-sensing system. This interaction, simulating a density-dependent motility effect, unexpectedly led to the sequential formation of highly periodic stripe patterns with temporal interval of $200 \mathrm{~min}$, after spotting the engineered strain on a semi-solid agar plate.

\section{Principle of pattern formation discovery by synthetic biology}

In Liu et al.'s study, they took the advantages of the 
synthetic system, in which the genetic circuit was designed, to measure every key quantity and quantitatively study the detailed dynamical process of stripe formation. This directly led to the proposal of densitydependent motility model. Unlike the other reactiondiffusion system, the density dependent motility system can initiate periodical stripes as the propagating wave front reaches the density threshold (Figure 3B). Besides, according to the model prediction, they found the system has a phase transition from stripes to non-stripes, between which finite numbers of stripes would be formed. To test this prediction in experiments, they succeeded to tune the number of stripes by changing the expression level of single gene. Detailed experimental and theoretical investigations of the pattern formation process led to the understanding of a new strategy of sequential stripe formation, which may be of relevance not only to the synthetic bacterial patterns but also to similar stripe formation processes in metazoan development.

\section{PERSPECTIVES}

Those who use traditional top-down research strategies to study biological pattern formation have to find a path through the complicated maze of cellular context and multi-layer regulation networks. In most cases, it's very difficult. Although in vitro bottom-up approaches can reconstitute some key players of certain biological systems, it is, after all, too artificial as well as requires detailed molecular mechanism(s). A more radical idea is to ignore the natural mechanisms, "create" biological pattern formation system, using synthetic biology, with phenotypes akin to existed ones. From these synthetic pattern systems, we may gain some new patterning principals. Given the vast diversity of natural patterns, it's unsurprising that analogous patterning systems exist. Researchers may benefit from revisiting their pattern systems with these "artificial" principals.

On the other hand, one of the goals of synthetic biology's is to build biological systems. We now understand many pieces, parts, and some of their interactions, but how to integrate them into a functional biologic system and ordered biological structure, e.g., blood vessel, skin, or even heart? It's a big challenge for tissue engineering. One approach may be $3 \mathrm{D}$ printing: directly build a "tissue" into its ultimate shape and size. The other way can be programmed development: starting from a cluster of cells, autonomously grow into a desired "tissue." For the latter one, it probably is more natural and durable. In the future, the combination of the two approaches could be joined to generate designed biological structures and to understand basic principles.

\section{COMPLIANCE WITH ETHICS GUIDELINES}

The authors Chenli Liu, Xiongfei Fu, and Jian-Dong Huang declare they have no conflict of interests.

This article does not contain any studies with human or animal subjects performed by any of the authors.

\section{REFERENCES}

1. Wolpert, L. (1969) Positional information and the spatial pattern of cellular differentiation. J. Theor. Biol., 25, 1-47

2. Danino, T., Mondragón-Palomino,O., Tsimring, L. and Hasty, J. (2010) A synchronized quorum of genetic clocks. Nature, 463, 326-330

3. Tabor, J. J., Salis, H. M., Simpson, Z. B., Chevalier, A. A., Levskaya, A., Marcotte, E. M., Voigt, C. A. and Ellington, A. D. (2009) A synthetic genetic edge detection program. Cell, 137, 1272-1281

4. Levskaya, A., Chevalier, A. A., Tabor, J. J., Simpson, Z. B., Lavery, L. A., Levy, M., Davidson, E. A., Scouras, A., Ellington, A. D., Marcotte, E. M., et al. (2005) Synthetic biology: engineering Escherichia coli to see light. Nature, 438, 441-442

5. Basu, S., Gerchman, Y., Collins, C. H., Arnold, F. H. and Weiss, R. (2005) A synthetic multicellular system for programmed pattern formation. Nature, 434, 1130-1134

6. You, L., Cox, R. S. 3rd, Weiss, R. and Arnold, F. H. (2004) Programmed population control by cell-cell communication and regulated killing. Nature, $428,868-871$

7. Elowitz, M. B. and Leibler, S. (2000) A synthetic oscillatory network of transcriptional regulators. Nature, 403, 335-338

8. Elowitz, M. and Lim, W. A. (2010) Build life to understand it. Nature, $468,889-890$

9. Mukherji, S. and van Oudenaarden, A. (2009) Synthetic biology: understanding biological design from synthetic circuits. Nat. Rev. Genet., 10, 859-871

10. Held, L. I. (1992) Models for Embryonic Periodicity. Basel; New York: Karger. viii, 119

11. Nüsslein-Volhard, C. and Wieschaus, E. (1980) Mutations affecting segment number and polarity in Drosophila. Nature, 287, 795-801

12. Lawrence, P. A. (1992) The Making of a Fly: the Genetics of Animal Design. Oxford: Blackwell Scientific Publications. xiii, 228

13. Peel, A. D., Chipman, A. D. and Akam, M. (2005) Arthropod segmentation: beyond the Drosophila paradigm. Nat. Rev. Genet., 6, 905-916

14. Dequéant, M. L. and Pourquié, O. (2008) Segmental patterning of the vertebrate embryonic axis. Nat. Rev. Genet., 9, 370-382

15. Schnell, S., Maini, P. K., McInerney, D., Gavaghan, D. J. and Houston, P. (2002) Models for pattern formation in somitogenesis: a marriage of cellular and molecular biology. C. R. Biol., 325, 179-189

16. Sadler, T. W. and Langman, J. (2000) Langman's Medical Embryology. Philadelphia: Lippincott Williams \& Wilkins. x, 504

17. Dubrulle, J., McGrew, M. J. and Pourquié, O. (2001) FGF signaling controls somite boundary position and regulates segmentation clock control of spatiotemporal Hox gene activation. Cell, 106, 219-232

18. Pourquié, O. (2003) The segmentation clock: converting embryonic time into spatial pattern. Science, 301, 328-330

19. Finney, M. and Ruvkun, G. (1990) The unc- 86 gene product couples cell lineage and cell identity in C. elegans. Cell, 63, 895-905 
20. McKearin, D. M. and Spradling, A. C. (1990) Bag-of-marbles: a Drosophila gene required to initiate both male and female gametogenesis. Genes Dev., 4, 2242-2251

21. Sussex, I. M. (1989) Developmental programming of the shoot meristem. Cell, 56, 225-229

22. Murray, J. D. (2002) Mathematical biology. New York: Springer.

23. Cooke, J. (1975) Control of somite number during morphogenesis of a vertebrate, Xenopus laevis. Nature, 254, 196-199

24. Alon, U. (2007) An Introduction to Systems Biology: Design Principles of Biological Circuits. Boca Raton, FL: Chapman \& Hall/CRC. xvi, 301, 304

25. May, R. M. (1976) Simple mathematical models with very complicated dynamics. Nature, 261, $459-467$

26. Wolpert, L. (1971) Positional information and pattern formation. Curr. Top. Dev. Biol., 6, 183-224

27. Kondo, S. and Miura, T. (2010) Reaction-diffusion model as a framework for understanding biological pattern formation. Science, $329,1616-1620$

28. Hodges, A. (1983) Alan Turing: The Enigma. New York: Simon and Schuster. 587

29. Turing, A. M. (1990) The chemical basis of morphogenesis. 1953. Bull. Math. Biol., 52, 153-197, discussion 119-152

30. Fitzhugh, R. (1961) Impulses and physiological states in theoretical models of nerve membrane. Biophys. J., 1, 445-466

31. Meinhardt, H. and Gierer, A. (1974) Applications of a theory of biological pattern formation based on lateral inhibition. J. Cell. Sci., 15, 321-346

32. Maini, P. K., Myerscough, M. R., Winters, K. H. and Murray, J. D. (1991) Bifurcating spatially heterogeneous solutions in a chemotaxis model for biological pattern generation. Bull. Math. Biol., 53, 701719

33. Swindale, N. V. (1980) A model for the formation of ocular dominance stripes. Proc. R. Soc. Lond., B, Biol. Sci., 208, 243-264

34. Murray, J. D., Oster, G. F. and Harris, A. K. (1983) A mechanical model for mesenchymal morphogenesis. J Math Biol, 17, 125-129

35. Murray, J. D. (2002) Mathematical Biology. II, Spatial Models and Biomedical Applications. New York: Springer. xxv, 811
36. Müller, P., Rogers, K. W., Jordan, B. M., Lee, J. S., Robson, D., Ramanathan, S. and Schier, A. F. (2012) Differential diffusivity of Nodal and Lefty underlies a reaction-diffusion patterning system. Science, 336, 721-724

37. Cates, M. E., Marenduzzo, D., Pagonabarraga, I. and Tailleur, J. (2010) Arrested phase separation in reproducing bacteria creates a generic route to pattern formation. Proc. Natl. Acad. Sci. U.S.A., 107, 1171511720

38. Fu, X., Tang, L. H., Liu, C., Huang, J. D., Hwa, T. and Lenz, P. (2012) Stripe formation in bacterial systems with density-suppressed motility. Phys. Rev. Lett., 108, 198102

39. Liu, C., Fu, X., Liu, L., Ren, X., Chau, C. K., Li, S., Xiang, L., Zeng, H., Chen, G., Tang, L. H., et al. (2011) Sequential establishment of stripe patterns in an expanding cell population. Science, 334, 238-241

40. Cooke, J. and Zeeman, E. C. (1976) A clock and wavefront model for control of the number of repeated structures during animal morphogenesis. J. Theor. Biol., 58, 455-476

41. Khalil, A. S. and Collins, J. J. (2010) Synthetic biology: applications come of age. Nat. Rev. Genet., 11, 367-379

42. Benner, S. A. and Sismour, A. M. (2005) Synthetic biology. Nat. Rev. Genet., 6, 533-543

43. Friedland, A. E., Lu, T. K., Wang, X., Shi, D., Church, G. and Collins, J. J. (2009) Synthetic gene networks that count. Science, 324, 1199-1202

44. Skerker, J. M., Perchuk, B. S., Siryaporn, A., Lubin, E. A., Ashenberg, O., Goulian, M. and Laub, M. T. (2008) Rewiring the specificity of twocomponent signal transduction systems. Cell, 133, 1043-1054

45. Kobayashi, H., Kaern, M., Araki, M., Chung, K., Gardner, T. S., Cantor, C. R. and Collins, J. J. (2004) Programmable cells: interfacing natural and engineered gene networks. Proc. Natl. Acad. Sci. U.S.A., 101, 8414-8419

46. Balagaddé, F. K., Song, H., Ozaki, J., Collins, C. H., Barnet, M., Amold, F. H., Quake, S. R. and You, L. (2008) A synthetic Escherichia coli predator-prey ecosystem. Mol. Syst. Biol., 4, 187

47. Lee, H. H., Molla, M. N., Cantor, C. R. and Collins, J. J. (2010) Bacterial charity work leads to population-wide resistance. Nature, 467, $82-85$ 